\title{
Monitoring der Sättigungszustände von Deichbauwerken mittels impedimetrischer Sensoren
}

\author{
Wolfgang Fichtner ${ }^{1}$, Michael Mertig ${ }^{1}$ Dirk Fleischer ${ }^{2}$ und Ulf Helbig ${ }^{2}$ \\ ${ }^{1}$ Kurt-Schwabe-Institut für Mess- und Sensortechnik Meinsberg e.V., Waldheim, Deutschland \\ ${ }^{2}$ Institut für Wasserbau und Technische Hydromechanik der Technischen Universität Dresden, Dresden, \\ Deutschland \\ Kontakt: wolfgang.fichtner@ksi-meinsberg.de
}

\section{Einleitung}

Auf der Grundlage der aktuellen Klimawirkungs- und Risikoanalyse des Umweltbundesamtes ist ein stark erhöhtes Risiko der Standsicherheit von Deichen und Dammbauwerken durch meteorologische Extremereignisse regionaldifferenzierter Ausprägung festzustellen [1]. Dies erfordert im Sinne der zivilen Sicherheit zwingend eine kritischere, prognostische Betrachtung der Stabilität von Deichbauwerken, aber auch möglicher Mechanismen zur Früherkennung von Einwirkungen, die über die bisherigen Bemessungswerte hinausgehen. Flankiert von heftigen Starkregenereignissen (erheblicher oberflächlicher Wasserandrang) nehmen umgekehrt auch lange heiße, niederschlagsarme Phasen und damit eine oberflächliche Austrocknung zu. Die feuchte- bzw. sättigungsabhängigen geotechnischen Eigenschaften sind wiederum für die dauerhafte Standsicherheit und Stabilität eines Deichbauwerks von entscheidender Bedeutung.

Kostengünstig herzustellende Sensoren ermöglichen, eingebettet in repräsentativen Zonen des Deiches, die Echtzeit-Überwachung von Sättigungszuständen und Wasserbewegungen im Erdbauwerk (Abb. 1).

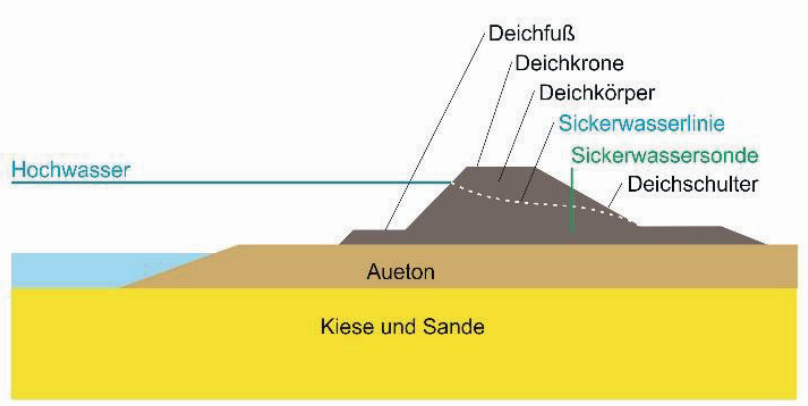

Abb. 1: Schematischer Deichquerschnitt bei hochwasserbedingtem, einseitigen Einstau.

Als Elektrodenmaterialien kommen Carbonfasermatten in spezieller, praxistauglicher Konfektionierung zum Einsatz, die für die Sanierung oder den Neuaufbau von Deichen geeignet sind. Die Entwicklung der Messtechnik am KSI Meinsberg wird von Untersuchungen begleitet, bei denen die Sensoren in Experimenten an einem realmaßstäblichen Versuchsdeich der TU Dresden mit vorgegebenen Einstauszenarien bis hin zum Deichversagen getestet werden [2]. Die Ergebnisse zeigen, dass impedimetrische Sensoren den Wassergehalt von Deichbaumaterialien qualitativ abbilden können. Die Praxis- und Langzeittauglichkeit der Sensoren ist mit Referenzmethoden zur Feuchtebestimmung verifiziert worden.

\section{Messmethodik}

\section{Impedimetrische Sensoren}

Das Sensorprinzip zur Bestimmung der Durchfeuchtung basiert auf der Impedanzmessung an einer Elektrodenanordnung im zu untersuchenden Bodenmaterial. Dabei kommen korrosionsfreie Carbonmatten zum Einsatz, die horizontal in das zu untersuchende Material eingebettet werden. Aufgrund ihrer offenen Gewebestruktur mit Spalten zwischen benachbarten Fasern und Lücken an Faserkreuzungen sind die Elektrodenmatten für auf- oder absteigende Feuchtigkeit durchlässig. Bei den Elektrodenanordnungen gemäß Abb. 2 ist das Volumen zwischen den Elektroden, wie auch der umgebende Raum mit dem zu untersuchenden Bodenmaterial gefüllt. Die Dreieranordnung der Elektroden (Abb. 2 unten) bietet u.a. den Vorteil, die vertikale Richtung der Feuchteänderung bestimmen zu können.
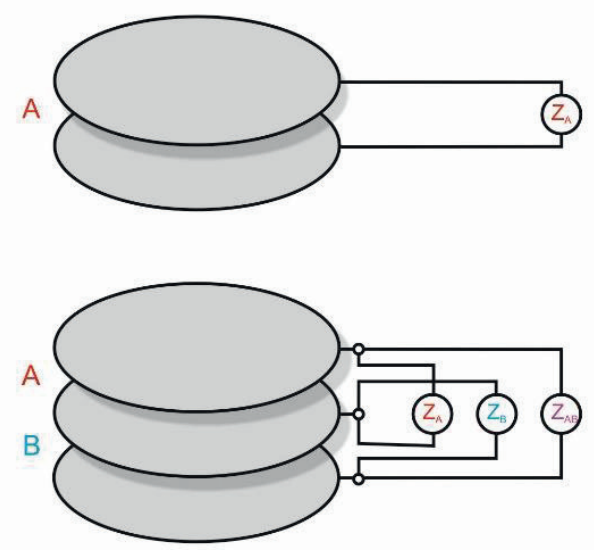

Abb. 2: Elektrodenanordnung zur Impedanzmessung, oben: Zweielektrodenanordnung, unten: Dreielektrodenanordnung.

Die Messung der Impedanzspektren erfolgte mit einem Potentiostaten Gamry IFC 1010 - 25019 im Frequenzbereich von $0,1 \mathrm{~Hz}$ bis $100 \mathrm{kHz}$. Die Verwendung eines elektrochemischen Multiplexer ECM-8 ermöglichte die quasi-simultane Messung (Programm: multiplexed potentiostatic eis repeating sequential) an acht Kanälen. 
Somit konnten in einer Laboranordnung bei Vorversuchen Langzeitmessungen mittels acht Messsensoren gleichzeitig durchgeführt werden.

Für den Einsatz in den verschiedenen Versuchsanordnungen des Projektes wurden Sensoren in Form von zylindrischen Elektrodenanordnungen aus Carbonmatten mit stabilem Ring und zugentlasteten Messkabeln konfektioniert (Abb. 3).

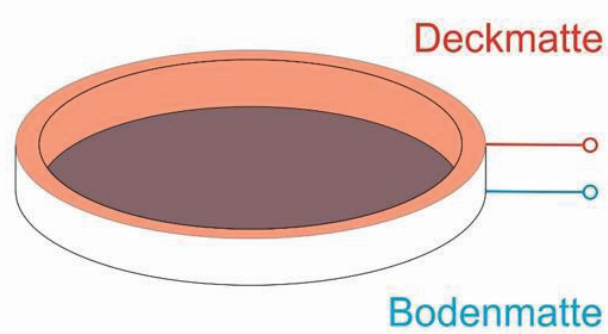

Abb. 3: Schematischer Aufbau eines impedimetrischen Feuchtesensors

Die Elektrodenmatten werden mit den Stirnflächen des Ringes verklebt und mit geschirmtem Doppelkoaxialkabel kontaktiert. Der Übergang der Kohlenstofffaser auf die Kupferlitze des Messkabels wird durch eine Klemmverbindung hergestellt. Diese ist gegen Einwirkung der Erdfeuchte isoliert angeordnet. Am Ring ist eine Kabelzugentlastung im Kunststoffmaterial angeschraubt und so der Sensor mechanisch robust gestaltet. Im Inneren ist der Sensor mit dem Mineralgemisch des vorgesehenen Einsatzortes gefüllt.

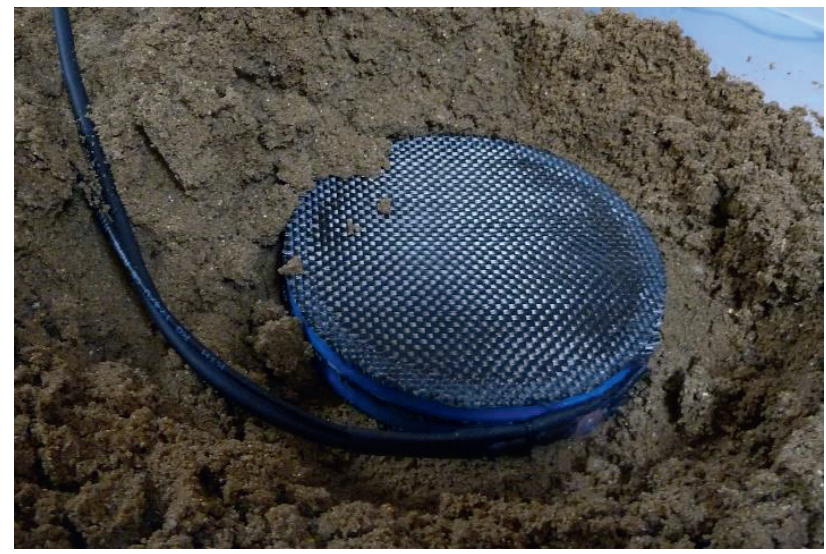

Abb. 4: Foto eines Feuchtesensors vor dem Einbau.

\section{Messaufbau Labor}

Um quantitative Rückschlüsse von den Messwerten zur Bodenfeuchte ziehen zu können ist die Kenntnis der Kalibrierfunktion erforderlich. Dazu sind in einem Laboraufbau Feuchtewerte in Sand von 2,5\% bis $20 \%$ (Masseanteil Wasser) vorgegeben, mehrere Sensoren in das gut homogenisierte Material eingebettet und verdichtet worden. Mit dem oben beschriebenen Versuchsaufbau zur Kalib- rierung lassen sich Impedanzwerte bei diskreten Feuchtewerten bestimmen.

Für den qualitativen Vergleich von Messverläufen mit den Werten eines Referenzsensors wurde ein Messaufbau nach Abb. 5 eingesetzt. Die Erfassung des Impedanzverlaufes an den beiden Feuchtesensoren a22 und a29 (Durchmesser $100 \mathrm{~mm}$ ) erfolgt durch Messung der Impedanz bei $1 \mathrm{kHz}$ mittels eines FLUKE PM 6306 (programmable automatic LCR-Meter). Die Referenzwerte werden mit kommerziell verfügbaren Bodenfeuchtesensoren EC-5 von METER group inc. USA mit einer Abtastrate von $1 \mathrm{~h}$ erfasst. Diese Langzeitversuche wurden in verschließbaren Probengefäßen durchgeführt, die mit ca. $15 \mathrm{dm}^{3}$ Rossendorfer Sand gefüllt sind. Dessen Durchlässigkeitswert $\mathrm{k}=1 \cdot 10^{-5} \mathrm{~m} / \mathrm{s}$ ermöglicht den Transport von Wasser innerhalb relativ kurzer Zeiträume, wodurch schnell veränderliche Durchfeuchtungszustände im Experiment gut nachgebildet werden können. Als Material für den Einsatz im realen Deichbau ist dieses Material aufgrund der hohen Durchlässigkeit weniger gut geeignet, kommt aber beispielsweise in Nordsachsen vor.

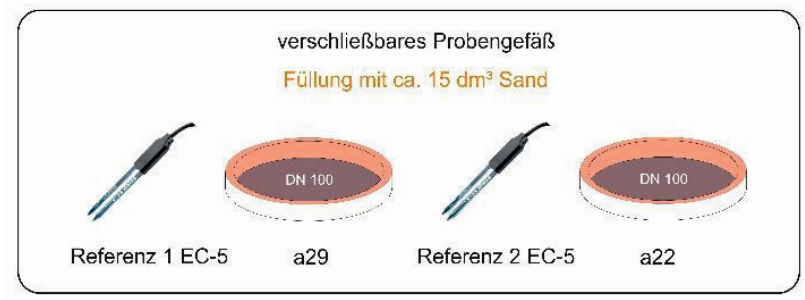

Abb. 5: Labormessanordnung zum Vergleich von Langzeitverhalten der Feuchtesensoren mit einem Referenzsensor EC-5.

\section{Messaufbau Versuchsdeich}

Zum Test der Funktionstüchtigkeit der Sensoren wurden 10 Exemplare in einen Versuchsdeich der Technischen Universität Dresden (TUD, Hubert-Engels-Labor, IWD) eingebaut. Der Deich besteht aus Sand (enggestuft, geringe Feinanteile), wodurch sich auch relativ schnell veränderliche hydrologische Szenarien vorgegeben werden können. Die Positionierung der Sensoren erfolgte in drei verschiedenen Ebenen (I, II und IV) gemäß Abb. 6.

Einige Sensoren wurden paarweise nebeneinander angeordnet, bspw. A13 und A4, um eine Dreidimensionalität der Deichdurchströmung untersuchen zu können. Die Einbaupositionen waren unterschiedlich weit von der Wasserseite entfernt ( $A 13$ und $A 14: 1 \mathrm{~m}, \mathrm{~A} 1, \mathrm{~A} 2, \mathrm{~A} 11, \mathrm{~A} 12$ und A15: 2,25 m sowie A3, A4 und A5: $4 \mathrm{~m}$ ). Die Deichhöhe beträgt mitsamt Bodenplatte $2 \mathrm{~m}$ bei einer Gesamtbreite am Deichfuß von $6 \mathrm{~m}$. Eine Ansicht der Messanordnung mit den Geräten zur Impedanzmessung auf der Krone des Versuchsdeiches ist in Abb. 7 dargestellt. Es wurden verschiedene Einstauszenarien von bis zu $96 \mathrm{~h}$ Dauer mit Scheitelhöhen des Wasserstandes von $95 \mathrm{~cm}$ und $120 \mathrm{~cm}$ untersucht. Die Impedanzspektren sind während der Einstauvorgänge an acht Kanälen mit einer Abtastrate von $1 \mathrm{~h}$ erfasst worden. 


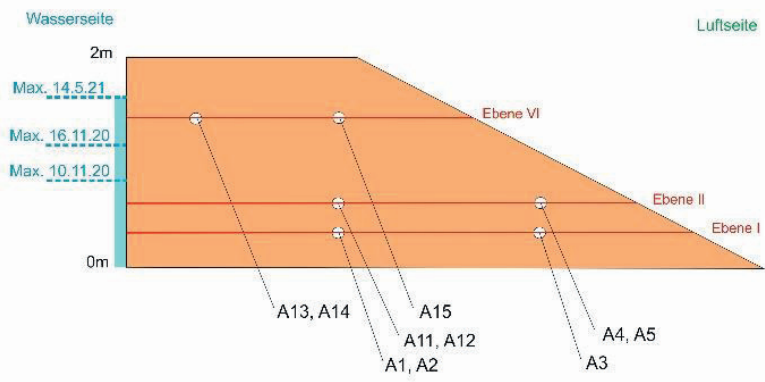

Abb. 6: Querschnitt durch einen Versuchsdeich der TUD mit Positionen der Sensoren A1 bis A15 (schematisch).

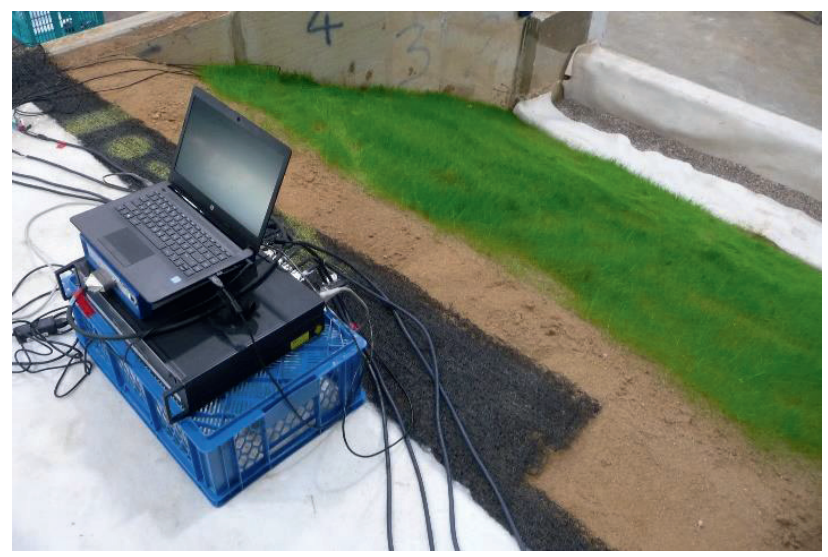

Abb. 7: Ansicht der Deichkrone in Richtung der mit Gras bewachsenen Luftseite des Versuchsdeichs der TUD mit Messequipment zur mehrkanaligen Impedanzmessung.

\section{Ergebnisse}

\section{Kalibrierung}

In Abb. 8 sind die Kalibrierkurven zweier Sensoren (DN $100 \mathrm{~mm}$ ) dargestellt. Die größte Sensitivität zeigt sich beim Betrag der Impedanz (Modulus) während die Phasenlage nur geringe Änderungen bei Variation der Feuchte aufweist. Dargestellt ist die Kalibrierkurve mit den bei 1 $\mathrm{kHz}$ gemessenen Impedanzwerten. Beide Kurven zeigen den erwarteten exponentiell abfallenden Verlauf. Die Absolutwerte der Impedanz der baugleichen Sensoren liegen auseinander, was auf eine Exemplarstreuung der einzelnen Sensoren hinweist. Einen großen Einfluss auf die Sensivität besitzt die Verdichtung des Füllmaterials der Sensoren und deren mechanischer Kontakt mit den Carbonelektroden. Zum gegenwärtigen Zeitpunkt sind noch nicht alle Ursachen für die Abweichungen vollständig bekannt und Gegenstand weiterer Untersuchungen.

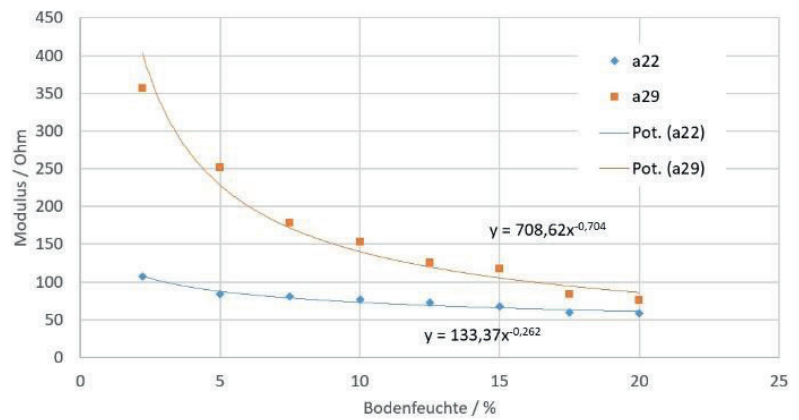

Durch Umrechnung der komplexen Impedanzwerte in die reziproke Größe Admittanz erhält man einen weitestgehend linearen Kurvenverlauf gemäß Abb. 9. Dieser ist in der Praxis mathematisch einfacher zu handhaben. Die unterschiedliche Lage und Steilheit der Kalibriergeraden werden wie bereits oben erwähnt durch Exemplarstreuungen der baugleichen Sensoren verursacht.

Abb. 8: Kalibrierkurve (Impedanzwerte) von zwei Feuchtesensoren.

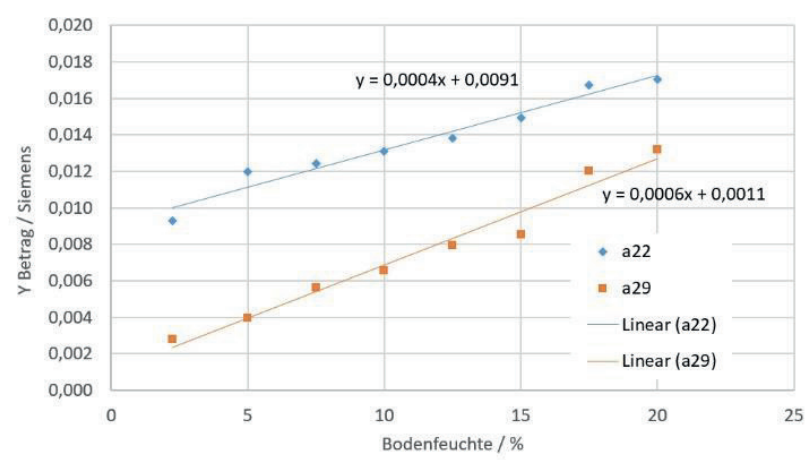

Abb. 9: Kalibrierkurve (Admittanzwerte) von zwei Feuchtesensoren.

\section{Temperaturverhalten}

Mit einer ähnlichen Versuchsanordnung wie bei den Kalibriermessungen wurde in Sand die Temperaturabhängigkeit den Feuchtesensoren bestimmt. Der Einsatz in der Praxis lässt stark schwankende Bodentemperaturen erwarten, deren Einfluss auf die Impedanzmessung hier quantifiziert werden soll. Dazu wurde die Anordnung im einem Klimaschrank im Bereich von 5 bis $50{ }^{\circ} \mathrm{C}$ bei einer Bodenfeuchte von 5 Masse- $\%$ temperiert.

Abb. 10 zeigt die Abhängigkeit zweier Sensoren von der Temperatur in Arrhenius'scher Darstellung. Dazu werden 
die logarithmierten Moduluswerte $(1 \mathrm{kHz})$ über der reziproken absoluten Temperatur aufgetragen.

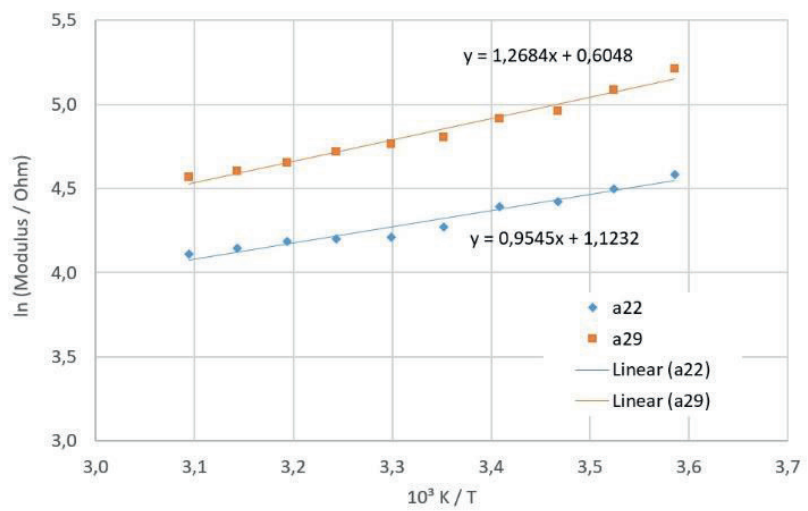

Abb. 10: Temperaturverhalten von zwei Sensoren a22 und a29 (Modulus bei $1 \mathrm{kHz}$ ).

\section{Referenzmessung im Langzeitversuch}

$\mathrm{Zu}$ Versuchsbeginn wurde eine Feuchtegehalt von 15 Masse- $\%$ in dem homogenisierten Bodenmaterial eingestellt. Danach schließt sich eine kurze Phase des Ausgleichs an, in der die Sensoren mit ihrer unmittelbaren Umgebung equilibrieren (Abb. 11). Dabei misst der Referenzsensor EC-5 1 einen Feuchtewert von ca. 20 Vol.-\% und der Referenzsensor EC-5 2 einen Feuchtewert von ca. 24 Vol.-\%. Zu beachten ist, dass sich der volumetrische Feuchtewert und der gravimetrische Feuchtewert durch den Proportionalitätsfaktor Trockendichte des untersuchten Materials unterscheiden. Der volumetrische Feuchtewert hängt somit direkt von der Verdichtung des Materials in der unmittelbaren Umgebung des Sensors ab. Unterschiede in der Verdichtung des Bodenmaterials bilden sich somit in den Messwerten ab und führen zu systematischen Abweichungen.

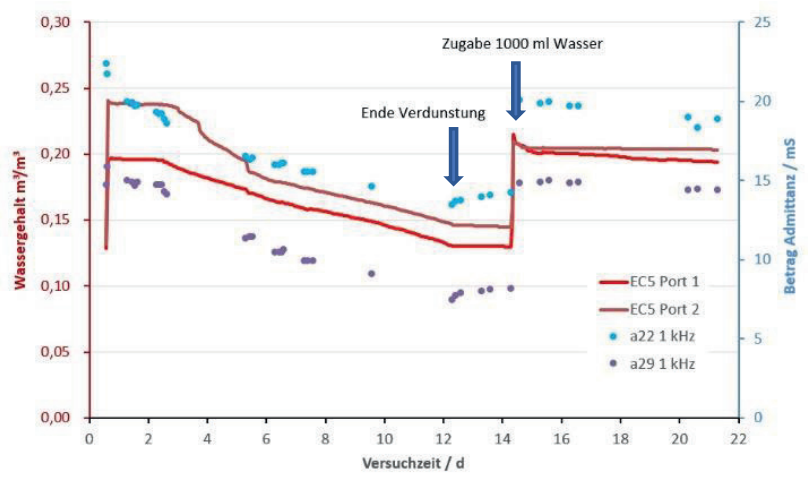

Abb. 11: Vergleich der Zeitreihen der Admittanz der Sensoren a22, a29 mit den Signalen der Referenzsensoren EC-5 1 und 2.

Wird die Anordnung geöffnet, so dass die Verdunstung von Wasser aus dem Bodenmaterial in die Umgebungsluft möglich ist, sinken die Feuchtewerte im Boden ab. Dies ist in den abfallenden Kurvenverläufen in Abb. $11 \mathrm{zu}$ be- obachten. Am Ende dieser Trocknungsphase liegt eine Feuchte von 8,9 Masse-\% vor. Die Referenzsensoren erfassen dabei Werte von 13 - 14 Vol.-\%. Der Betrag der Admittanz der Sensoren folgt tendenziell dem oben beschriebenen Kurvenverlauf. Am 12. Versuchstag wurde die Versuchsanordnung verschlossen und damit die Verdunstung in die Umgebungsluft beendet. Die Referenzsensoren gehen ab diesem Zeitpunkt in eine Phase mit konstanten Werten über. Die Admit-tanzwerte der Sensoren a22 und a29 steigen leicht an, wahrscheinlich diffundiert Feuchtigkeit aus den unter den Sensoren angrenzenden Bereichen in die Sensorfüllung ein und führt so erst nach ca. $48 \mathrm{~h}$ zu konstanten Werten. Um wieder auf den Ausgangswert von 15 Masse-\% Feuchte zu kommen, wurde am 14. Versuchstag $1000 \mathrm{ml}$ Wasser verteilt über die Sandoberfläche in die Versuchsanordnung gegeben. In dem sich anschließenden Zeitfenster mit abgedeckter Anordnung ohne Verdunstung stellen sich nach einer kurzen Ausgleichsphase wieder konstante Kurvenverläufe für die Referenz wie auch die Admittanz ein. Insgesamt kann aus dem Vergleich der Kurvenverläufe eine gute Übereinstimmung bei der Wiedergabe der qualitativen Tendenzen in den unterschiedlichen Versuchsphasen festgestellt werden.

\section{Versuchsdeich}

Die in den Versuchsdeich eingebauten Sensoren liefern im Abstand von $1 \mathrm{~h}$ ein komplettes Impedanzspektrum im Bereich von $0,1 \mathrm{~Hz}$ bis $100 \mathrm{kHz}$. In Abb. 12 sind die daraus bestimmten Admittanzverläufe (Modulus) des Sensors A3 während eines Anstauvorgangs über einen Zeitraum von $100 \mathrm{~h}$ dargestellt. Zur besseren Übersichtlichkeit wurde pro Frequenzdekade nur ein Admittanzverlauf aufgetragen. Der Sensor A3 befindet sich in der unteren Ebene I im Versuchsdamm (Abb. 6) und wird durch den Anstauvorgang vom teil- in den vollgesättigten Bereich überführt.

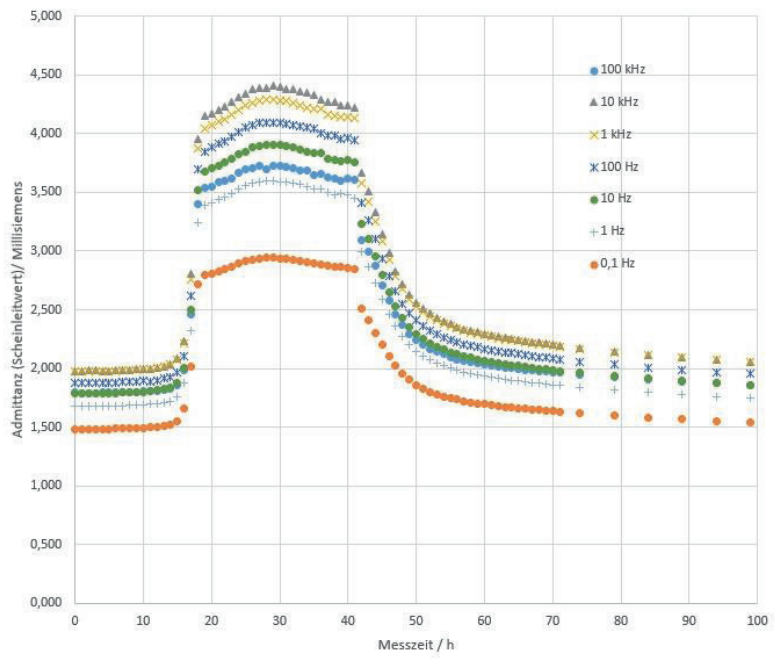

Abb. 12: Admittanzverläufe des Feuchtesensors A3, Anstau bis $\max .95 \mathrm{~cm}$. 
Die kommende Anstauwelle macht sich zunächst durch einen allmählichen Anstieg der Admittanz bemerkbar. Nach 15 h kommt es zu einem steilen Kurvenanstieg mit einer Verdoppelung der Leitwerte. Danach tritt eine Plateauphase ein, die den vollgesättigten Bereich für Sensor A3 markiert. In dieser Phase bis $40 \mathrm{~h}$ treten nur geringe Veränderungen der Admittanz auf, die vermutlich aus Effekten im Zusammenhang mit einem weiteren Anstieg des hydrostatischen Drucks resultieren. Der Abfall der Kurven erfolgt nach den Plateaus, also im Zeitfenster sinkender Durchfeuchtung, deutlich flacher. Offensichtlich kann erwartungsgemäß die Wasseraufnahme im Sand schneller erfolgen als die Wasserabgabe. Die Kurvenverläufe nähern sich nach dem Einstauvorgang asymptotisch den Ausgangswerten wieder an. Qualitativ zeigen die Kurven für alle betrachteten Messfrequenzen gleiche Verläufe. Im Folgenden sollen daher nur noch die Veränderungen bei einer Arbeitsfrequenz von $1 \mathrm{kHz}$ betrachtet werden.

Ein weiterer Versuch wurde mit einer Ganglinie durchgeführt, bei der der maximale Einstaupegel bei ca. $165 \mathrm{~cm}$ lag (Abb. 13). Das Plateau des Pegelverlaufs zwischen 90 $\mathrm{h}$ und $137 \mathrm{~h}$ wurde durch einen kurzen Abfall unterbrochen.

Der Vergleich von Admittanzverläufen von acht Sensoren, die in unterschiedlichen Bereichen des Versuchsdeiches positioniert sind, ist Abb. $14 \mathrm{zu}$ entnehmen. Die Zeitachsen in beiden Darstellungen Abb. 13 und 14 wurden synchronisiert. Die Sensoren A1 und A3 befindet sich in der unteren Ebene und werden bereits nach 40 h bzw. $50 \mathrm{~h}$ von der ansteigenden Sickerlinie überstrichen. Danach befinden sie sich im vollgesättigten Bereich des Deiches. Die oberhalb Die in Ebene II angeordneten Sensoren A4 und A5 (Abb. 6) gehen erst nach ca. $80 \mathrm{~h}$ in den vollgesättigten Bereich über. Die Admittanzkurven der Sensoren A11 und A12 steigen nach $75 \mathrm{~h}$ an und erreichen die Plateauphase nach ca. 87 h. Nach 120 h zeigen beide Sensoren den kurzzeitigen Rückgang der Admittanzwerte, wie er durch den Pegelrückgang in Abb. 13 zu erwarten war.

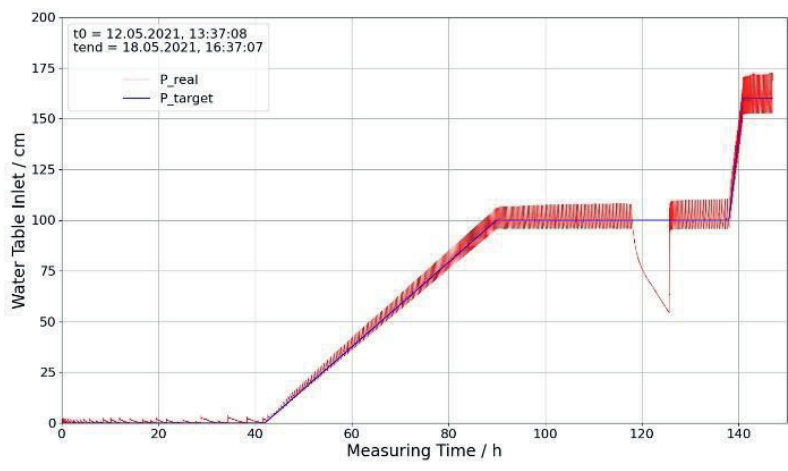

Abb. 13: Pegelverlauf am Versuchsdeich, Anstau auf der Wasserseite bis max. $165 \mathrm{~cm}$.
Die in Ebene IV angeordneten Sensoren A13 und A15 werden vom Einstau erst nach ca. $138 \mathrm{~h}$ beeinflusst. Der zugehörige Kurvenverlauf zeigt einen sehr steilen und hohen Anstieg, der bei A15 nach kurzer Zeit (ca. 6 h) wieder abfällt. Die Kurvenform mit dem fehlenden Plateau lässt den Schluss zu, dass sich der Sensor im teilgesättigten Bereich befindet. Der näher an der Wasserseite positionierte Sensor A13 zeigt ein kurzes Plateau, was darauf schließen lässt, dass er sich während dieser Zeit in der gesättigten Zone befand.

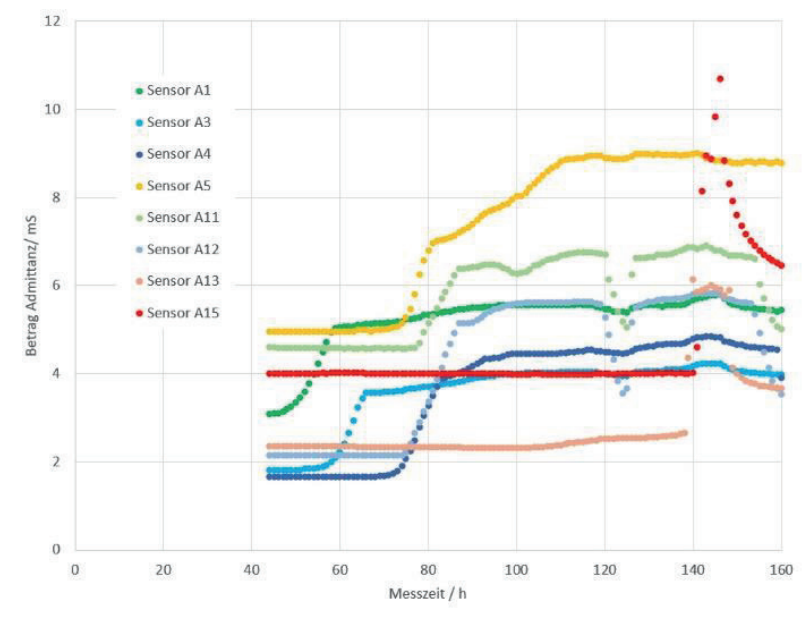

Abb. 14: Admittanzverläufe der Feuchtesensoren A3, A5 und A15, Anstau $165 \mathrm{~cm}$.

\section{Diskussion}

Vor dem Hintergrund sich wandelnder klimatischer Einflüsse nimmt die Wahrscheinlichkeit längerer Trockenperioden und von Starkregenereignissen in Mitteldeutschland zu. Gerade die Kombination beider Effekte mit einhergehender Riss- und Kluftbildung infolge Austrocknung der Deichstruktur und darauffolgender schneller Durchnässung führen zu kritischen Zuständen hinsichtlich Standsicherheit, Lebensdauer und Dichtheit von Deichen. Werkzeuge zur strukturellen Analyse in Echtzeit, wie automatisierte Bereitstellung von Versagenswahrscheinlichkeiten basierend auf Sensornetzwerken und effizienter Interpretation durch mathematische Modelle, sind essenziell für Hochwasserschutzanlagen der Zukunft [3]. Eine Implementierung in technische Regelwerke steht bisher aber noch aus.

Der Einsatz preisgünstiger impedimetrischer Sensoren integriert in Monitoringsysteme zur Versagenswahrscheinlichkeit von Deichbauwerken, kann bei weiterer Verbesserung der sensorischen Eigenschaften, insbesondere hinsichtlich der Erfassung absoluter Feuchtewerte für künftige Applikationen an kritischen Deichabschnitten in Sachsen zur technischen Option werden. 


\section{Literatur}

[1] UMWELTBUNDESAMT (Hg.) (2021): Klimawirkungsund Risikoanalyse 2021 für Deutschland. Teilbericht 6: Integrierte Auswertung - Klimarisiken, Handlungserfordernisse und Forschungsbedarfe. Unter Mitarbeit von Adelphi Research, Eurac Research und Bosch \& Partner GmbH. Dessau-Roßlau.

[2] FICHTNER, W.; MERTIG, M.; FLEISCHER, D.; HELBIG, U.: Impedimetrische Sensoren zur Überwachung von Deichbauwerken. 26. Interdisziplinäre Wissenschaftliche Konferenz Mittweida (2021) 25-28, DOI: 10.48446/opus-12301.

[3] PONZIANI, M., BACHMANN, D.: Real-Time Monitoring and Forecasting of Dike Strength. Int. J. of Safety and Security Eng., Vol. 6, No. 2 (2016) 122-131.

\section{Danksagung}

Die vorgestellten Arbeiten sind Teil eines Projektes gefördert vom Europäischen Fond für Regionale Entwicklung (EFRE), FKZ 100362350.

Die Autoren bedanken sich bei der SAB für die finanzielle Unterstützung sowie den Projektpartnern:

M\&S Umweltprojekt GmbH, Plauen,

Institut für Grundwasser, TU Dresden, Dresden, und

Weischlitzer Tiefbau und Umweltschutz GmbH, Weischlitz,

für die angenehme Zusammenarbeit. 